Article

\title{
Research on Control Technologies for a High-Precision Multi-Source Vibration Simulation System
}

\author{
Xibin Ma ${ }^{1, *}$, Zhangwei Chen ${ }^{1}$, Huinong $\mathrm{He}^{2}$ and Yugang Zhao ${ }^{2}$ \\ 1 The State Key Lab of Fluid Power Transmission and Control, Zhejiang University, Hangzhou 310027, China; \\ chenzw@zju.edu.cn \\ 2 Hangzhou ECON Science and Technology Co., Ltd., Hangzhou 310015, China; \\ huinonghe@econ-group.com (H.H.); yugangzhao@econ-group.com (Y.Z.) \\ * Correspondence: $11425029 @ z j u . e d u . c n$
}

Received: 28 September 2018; Accepted: 23 October 2018; Published: 29 October 2018

\begin{abstract}
Vehicles commonly suffer from the narrow-band noises and vibrations, usually a superposition of multiple sinusoidal signals, due to the excitations of engines, electrical motors, gear boxes, and other rotating mechanical parts. These excitations are transmitted to a reference point of some structure with certain transmission paths. The vibration signal measured at the reference point can be used for power system monitoring, fault diagnosis, modal analysis, noise analysis, etc. For convenience, researchers in a laboratory usually use shakers to generate expected narrow-band vibration signals acting on the vehicle structure reference points to simulate the vibration signals. However, there is a prominent difficulty in ensuring the amplitude and phase accuracy of each sub-frequency component simultaneously. In order to improve the accuracy of generating the expected vibration signal, this paper presents a multi-source vibration simulation control technology based on the tracking filter method. The main idea is to use the tracking filter to estimate the amplitude and phase of the target sub-frequency component accurately. Further, on the target sub-frequency, the drive signal of shakers is then corrected based on the amplitude and phase errors to achieve a more accurate target vibration signal. The amplitude and phase of each sub-frequency component in the excitation signal can be controlled independently. Compared with other Fast Fourier Transform (FFT)-based frequency domain analysis algorithms and numerical methods by solving the equations, the tracking filter method has a higher frequency resolution and higher accuracy. It can be easily realized in real time applications due to its simplicity. Finally, verification experiments are completed. The experimental results show that the multi-source vibration simulation control technology presented in this paper can achieve high-precision amplitude and phase on each sub-frequency component of the target vibration signals, which contain up to eight sub-frequency components.
\end{abstract}

Keywords: multi-source vibration simulation; vehicle noise control; high precision vibration reproduction

\section{Introduction}

Engines and electrical motors are the core components of vehicles' power systems. Together with gear boxes and other rotating mechanical parts in the kinematic chains, they produce vibration forces impacting the vehicle's vibration and noise environment [1-4]. Furthermore, the vibration may also impact the structure life-time, component reliability, vehicle comfort, etc. The topics regarding collecting and analyzing the vibration and noise signals at some reference points for faulty diagnosis [2-4], power system health monitoring [5,6], structure modal analysis [7-11], and noise and 
vibration control [12-14] have been popular for decades. From this literature, it is also shown that in some circumstances of faulty diagnosis and noise control, the key information contained in the vibration and noise signals are presented as some special combinations of sinusoidal signals. These vibration and noise signals are always collected under working condition, so it may be not convenient to get them handily.

For experimental research, a widely used solution in the laboratory is using shakers to generate target narrow-band vibration signals acting on the reference points to simulate the expected vibration and noise conditions. Obviously, a single frequency sinusoidal excitation cannot meet the requirements of the research like structure modal analysis and noise control mentioned above, which need multi-frequency excitations. Installing multiple shakers may affect the automobile structural modal and introduce analysis error. Sometimes the limited space of a vehicle's structure will restrict the use of multiple shakers. Therefore, researchers prefer to use a single shaker to generate a composite excitation containing multiple frequency components. However, due to factors such as the complicated structural modal, the characteristics of the shaker (such as time-varying transfer function), and the varying working conditions (such as shifting), it is difficult to achieve high-precision control of the amplitude and phase of each frequency component.

A typical control schematic for producing narrow-band excitation $[15,16]$ is shown in Figure 1, which has also been widely used in vibration test systems. The control error is obtained by comparing the characteristic parameters of the response signal with the control target value in a real-time closed control loop, and then the drive signal is updated using the error signal. Many algorithms have been studied for the purpose of amplitude and phase estimation. Fast Fourier Transform (FFT)-based estimation algorithms are widely used to estimate the frequency, amplitude and, phase of sinusoidal signals. The estimation accuracy and frequency resolution are closely related to points of samples. Using numerical methods like solving equations directly has higher frequency resolution and requires fewer data points [17-20]. However, numerical methods need to calculate the inverse of matrix and carefully handle some ill-conditions in common. When the number of sub-frequencies is big, the amount of calculations will grow very fast [21,22]. Correlation functions can also easily estimate amplitudes and frequencies of periodic signals, but the phase information will be lost [23,24]. It is worth mentioning that some Least-Mean-Square (LMS)-based algorithms like Active Noise Equalization Algorithm (ANE) and Enhanced Active Noise Equalization Algorithm (EANE) could be used to manipulate multi-frequency narrow-band excitations and achieve very high accuracy [25-27]. Yet, researchers must pay more attention to those LMS-based algorithms for balancing the control accuracy and convergence rate, especially in frequency-varying applications. All these algorithms must be designed properly for real-time applications.

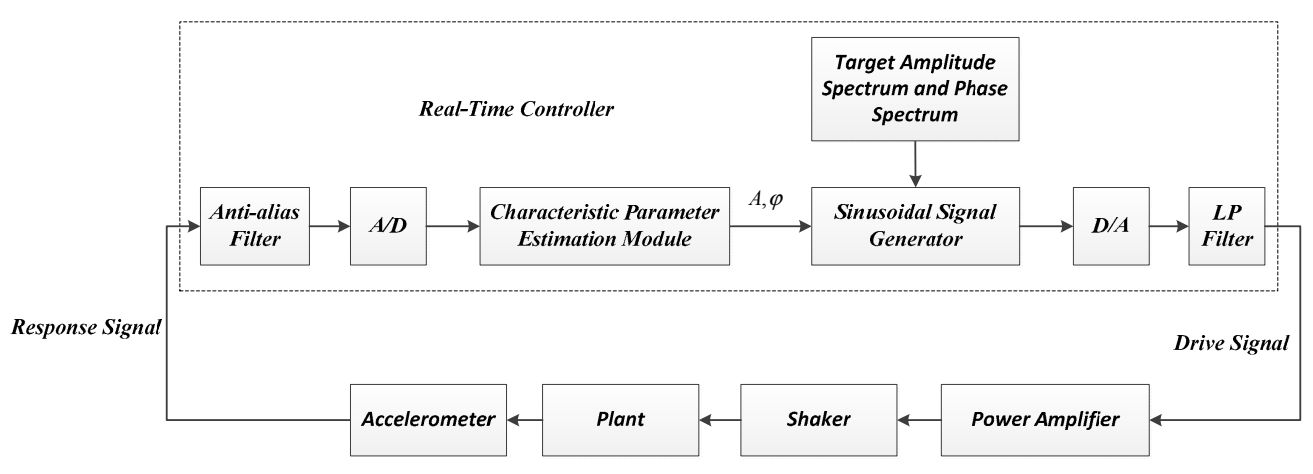

Figure 1. The control schematic for producing narrow-band excitation.

In order to meet the requirements of high-precision multi-source vibration simulation, this paper studied some technologies including drive synthesis methods based on explicit frequency-varied sinusoidal calculating algorithms and multi-frequency characteristic parameter estimation algorithms based on parallel tracking filters. The drive synthesis methods can improve the unsteady state 
compared with other phase-recursive methods. The parallel tracking filters are used to estimate each frequency component's amplitude and phase independently, which yields high frequency resolution and effective reduction of the mutual influence between frequency components [15]. All of these technologies are combined to achieve a high-precision control result for each frequency component's amplitude and phase.

\section{Drive Synthesis for Multi-Source Excitation}

There is a concealed unsteady state phenomenon in program design, typically, caused by the accumulation of truncations, rounding errors, and the finite word length effect. It will ruin the phase information of a sinusoidal signal when the control program runs for a long time. In this paper, the sinusoidal drive signal is calculated explicitly by solving the phase differential equation $[10,15]$. The drive signal containing $\mathrm{N}$ frequency components is represented as follows:

$$
\mathrm{D}(\mathrm{t})=\sum_{i=0}^{N-1} A_{i} \sin \left[\theta_{i}(\mathrm{t})\right]
$$

$A_{i}$ and $\varphi_{i}$ are the amplitude and phase of the $i$-th frequency component, respectively. The instantaneous output frequency is the first derivative of the phase and the frequency conversion rate is the second derivative of the phase. Assuming the frequency $f_{i}$ is varying at a linear rate of $v_{i}$, the following two equations can be written:

$$
\begin{gathered}
\frac{\partial \theta_{i}(\mathrm{t})}{\partial \mathrm{t}}=2 \pi f_{i} \\
\frac{\partial^{2} \theta_{i}(t)}{\partial \mathrm{t}^{2}}=2 \pi \frac{\partial f_{i}}{\partial t}=2 \pi v_{i}
\end{gathered}
$$

By solving the above formulas, the phase calculation formula can be drawn:

$$
\theta_{i}(t)=2 \pi\left(\frac{v_{i} t^{2}}{2}+f_{i 0} t\right)+\theta_{i 0}
$$

where $f_{i 0}$ represents the initial frequency and $\theta_{i 0}$ is the initial phase. Obviously, $v_{i}=$ constant means that $i$-th component is a linear sweeping signal. If $v_{i}$ is a positive value, $f_{i}$ sweeps up. A negative $v_{i}$ means sweeping down. When $v_{i}=0, f_{i}$ is a constant value (dwell).

If frequency $f_{i}$ varies at a logarithmic rate, then:

$$
\begin{gathered}
f_{i}=f_{i 0} v_{i}{ }^{t} \\
\frac{\partial \theta_{i}(t)}{\partial \mathrm{t}^{2}}=2 \pi f_{i}=2 \pi f_{i 0} v_{i}{ }^{t}
\end{gathered}
$$

By solving the above formulas, the phase calculation formula can be drawn:

$$
\theta_{i}(t)=2 \pi f_{i 0}\left(\frac{v_{i}^{t}-1}{\ln v_{i}}\right)+\theta_{i 0}
$$

Here, if $v_{i}$ is positive, $f_{i}$ sweeps up at a logarithmic rate, and if $v_{i}$ is negative, $f_{i}$ sweeps down. When $v_{i}=0, f_{i}$ is constant. Then the phase of the $f_{i}$ component is calculated as follows:

$$
\theta_{i}(t)=2 \pi f_{i} t+\theta_{i 0}
$$

By using Equations (4), (7), and (8), the drive signal can be explicitly calculated point-by-point in an analytical way. Compared to the look-up table and phase iterative calculating algorithms, it can significantly improve the unsteady state problem of the drive signal. 


\section{Estimation Algorithms for Amplitude and Phase}

The tracking filter algorithm is derived from digital phase-locked loop method. It has certain applications in communication and vibration engineering for its advantages on high frequency resolution, efficiency and simplicity $[15,16]$. A parallel structure of digital tracking filters is shown in Figure 2. As the tracking filters are independent and the calculating procedures are identical, the parallel structure can be easily instantiated using multi-core processing systems or multi-threading processing technologies to estimate the amplitude and phase of all frequency components synchronously.

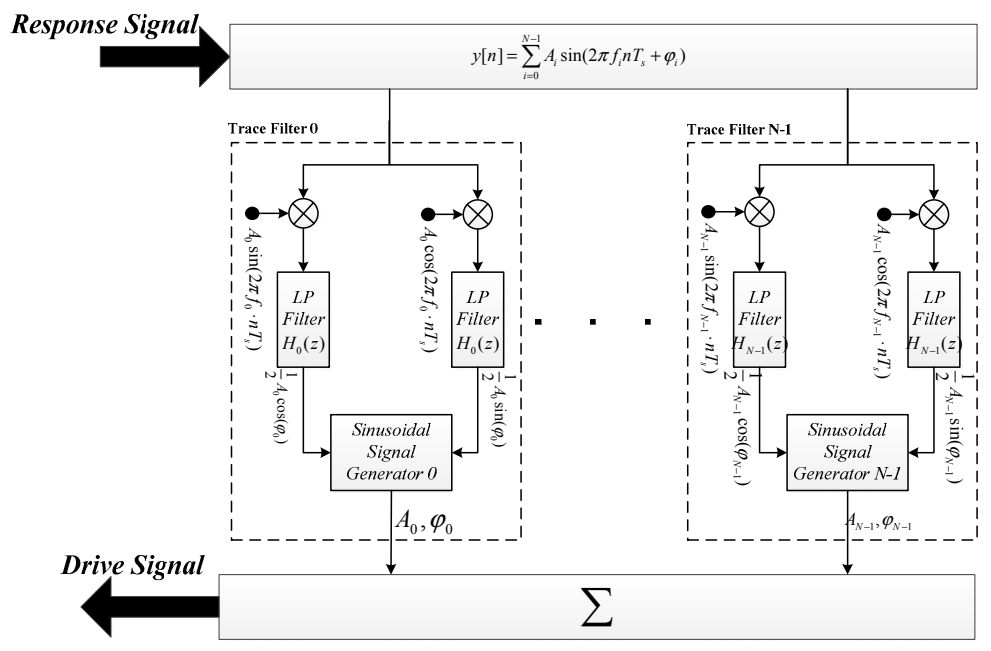

Figure 2. The parallel structure of digital tracking filters.

It should be noted that the adjacent frequencies have a mutual interference effect with each other when the tracking filter algorithm is applied. Unlike other control strategies that use fixed cutoff frequencies or optimal multi-fixed cutoff frequencies [10], this paper represents a strategy to update the cutoff frequencies dynamically. The cutoff frequency can be set to a fixed percentage value of the target frequency component or set to some special values according to working conditions. It is useful for balancing the response time of the tracking filters and the target frequency variation rules that researchers have set up. It also has an effect on suppressing the interference between adjacent frequencies. Taking the control procedure of the $j$ th target frequency component $f_{j}$ as an example, a processing flowchart can be drawn as Figure 3, and the details that amplitude control procedure using the tracking filtering algorithm can be summarized as follows:

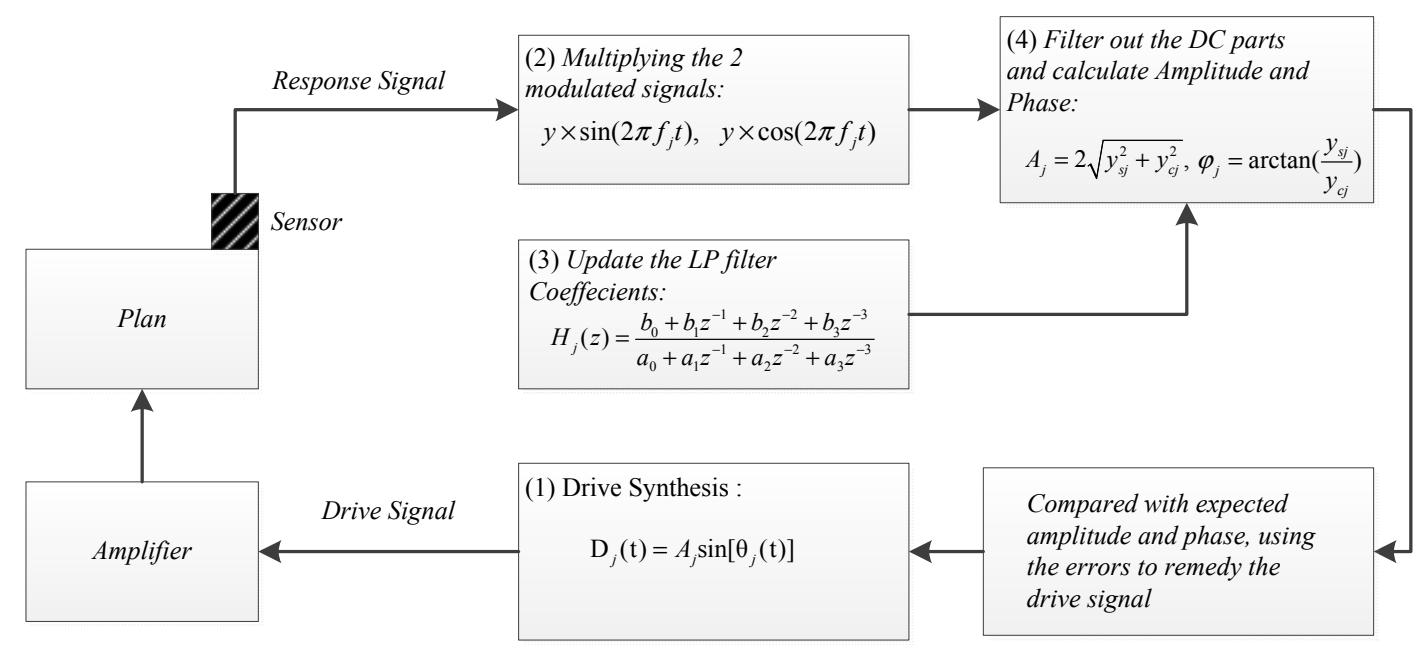

Figure 3. The flowchart of branch $\mathrm{j}$ in parallel tracking filters. 
(1) Using the sinusoidal generation method mentioned in Section 1 to generate a sine and a cosine modulated signal with the same frequency as the target frequency $f_{j}: A_{j} \sin \left(2 \pi f_{j} \mathrm{t}\right)$ and $A_{j} \cos \left(2 \pi f_{j} \mathrm{t}\right)$;

(2) Multiplying the response signal $y$ by the two modulated signals, using the trigonometric functions to derive the two equations below:

$$
\begin{gathered}
y \times \sin \left(2 \pi f_{j} t\right)=\sum_{i=0, i \neq j}^{N-1}\left\{\frac{1}{2} A_{j}\left[\cos \left(2 \pi\left(f_{i}-f_{j}\right) t+\varphi_{i}\right)-\cos \left(2 \pi\left(f_{i}+f_{j}\right) t+\varphi_{i}\right)\right]\right\} \\
-\frac{1}{2} A_{j} \cos \left(4 \pi f_{j} t+\varphi_{j}\right)+\frac{1}{2} A_{j} \cos \left(\varphi_{j}\right) \\
\left.y \times \cos \left(2 \pi f_{j} t\right)=\sum_{\substack{i=0, i \neq j \\
N-1}}^{N-1} A_{j}\left[\sin \left(2 \pi\left(f_{i}-f_{j}\right) t+\varphi_{i}\right)+\sin \left(2 \pi\left(f_{i}+f_{j}\right) t+\varphi_{i}\right)\right]\right\} \\
\quad+\frac{1}{2} A_{j} \sin \left(4 \pi f_{j} t+\varphi_{j}\right)+\frac{1}{2} A_{j} \sin \left(\varphi_{j}\right)
\end{gathered}
$$

From Equations (9) and (10), the result of multiplication contains a DC component, an AC component of two times the target frequency, and AC signals produced by the frequency components that differ from the target frequency. The DC components preserve the amplitude and phase information. A low-pass filter is used to extract those DC components from Equations (9) and (10).

(3) A three-order normalized Butterworth filter has been selected as the low-pass filter in the tracking filter algorithm:

$$
H_{j}(s)=\frac{1}{s^{3}+2 s^{2}+2 s+1}
$$

Let $F_{c j}=c_{j} \times f_{j}$ be the cutoff frequency of $H_{j}(s)$, where $c_{j}$ is the low-pass filter cutoff frequency factor of the target frequency $f_{j}$ and $f_{s}$ is sample frequency. The circular frequency $\Omega_{j}$ is:

$$
\Omega_{j}=\tan \left(\frac{2 \pi F_{c j}}{2}\right)=\tan \left(\frac{\pi c_{j} f_{j}}{f_{s}}\right)
$$

Performing the denormalization:

$$
H_{j}(s)=\frac{1}{\left(\frac{s}{\Omega_{j}}\right)^{3}+2\left(\frac{s}{\Omega_{j}}\right)^{2}+2\left(\frac{s}{\Omega_{j}}\right)+1}
$$

By using a bilinear transformation method, set $\mathrm{s}=\frac{1-z^{-1}}{1+z^{-1}}$, then the discrete form of the LP filter is:

$$
H_{j}(z)=\frac{b_{0}+b_{1} z^{-1}+b_{2} z^{-2}+b_{3} z^{-3}}{a_{0}+a_{1} z^{-1}+a_{2} z^{-2}+a_{3} z^{-3}}
$$

The coefficients of $H_{j}(z)$ are shown as follows:

$$
\left\{\begin{array}{l}
b_{0}=\Omega_{j}^{3}, b_{1}=3 \Omega_{j}^{3}, b_{2}=3 \Omega_{j}^{3}, b_{3}=\Omega_{j}^{3} \\
a_{0}=1+2 \Omega_{j}+2 \Omega_{j}^{2}+\Omega_{j}^{3}, a_{1}=-3-2 \Omega_{j}+2 \Omega_{j}^{2}+3 \Omega_{j}^{3} \\
a_{2}=3-2 \Omega_{j}-2 \Omega_{j}^{2}+3 \Omega_{j}^{3}, a_{4}=-1+2 \Omega_{j}-2 \Omega_{j}^{2}+3 \Omega_{j}^{3}
\end{array}\right.
$$

Then the low-pass filter coefficients can be updated using the above formula dynamically according to the first parts of Equations (9) and (10), which represent the mutual interference in tracking filters.

(4) Then, after the LP filter processing, the DC components are obtained:

$$
y_{s j}=\frac{1}{2} A_{j} \sin \left(\varphi_{j}\right), y_{c j}=\frac{1}{2} A_{j} \cos \left(\varphi_{j}\right)
$$


$A_{j}$ and $\varphi_{j}$ can be calculated as following:

$$
A_{j}=2 \sqrt{y_{s j}^{2}+y_{c j}^{2}}, \varphi_{j}=\arctan \left(\frac{y_{s j}}{y_{c j}}\right)
$$

\section{Experiments}

The experimental setup of the multi-source vibration simulation is shown in Figure 4. The hardware consists of the LT-2 integrated exciter with a bandwidth of $2 \sim 10,000 \mathrm{~Hz}$, the ECON VT-9008 vibration real-time controller, an acceleration sensor with the sensitivity of $10.6 \mathrm{mv} /\left(\mathrm{m} / \mathrm{s}^{2}\right)$, and a PC. ECON VCStest software was used for the parameter settings, curve display, and data analysis.

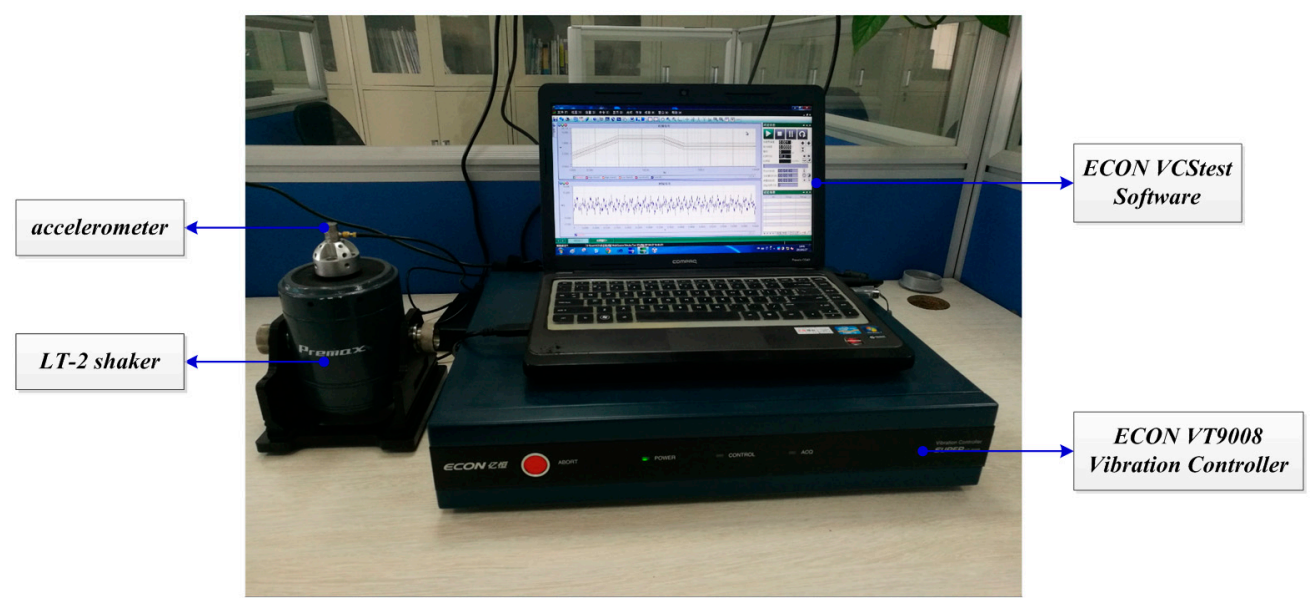

Figure 4. The experimental devices.

As an application of the algorithms presented in this paper, the Segmented-Sweep test is selected. Dwell (stable-frequency) excitation and logarithmic sweeping excitation are the most-used stimuli in automotive structural modal analysis and noise research. These two kinds of excitation experiments are selected to verify the effectiveness. Since Segmented-Sweep tests concern amplitude and frequency control results, the phase is not dropped here. In fact, using Equation (17), amplitude and phase values can be easily obtained simultaneously.

\subsection{The Dwell Experiment of Composite Excitation}

The spectrum setup is shown in Figure 5. In the frequency band $5 \sim 10,000 \mathrm{~Hz}$, eight frequency components were chosen for the dwell experiment (as shown in Table 1). The target amplitude of each frequency can be modified by setting the percent value compared to the spectrum.

The results of dwell experiment are shown in Figure 6. The exact amplitude values of each frequency component are shown in Table 1. The instantaneous relative control errors of each frequency component are $0.39 \%,-0.76 \%,-3.2 \%, 0.23 \%, 0.68 \%, 0.4 \%, 0.8 \%$, and $0.6 \%$, respectively. The experimental results show that the high-precision composite excitation containing up to eight frequency components has been achieved. 


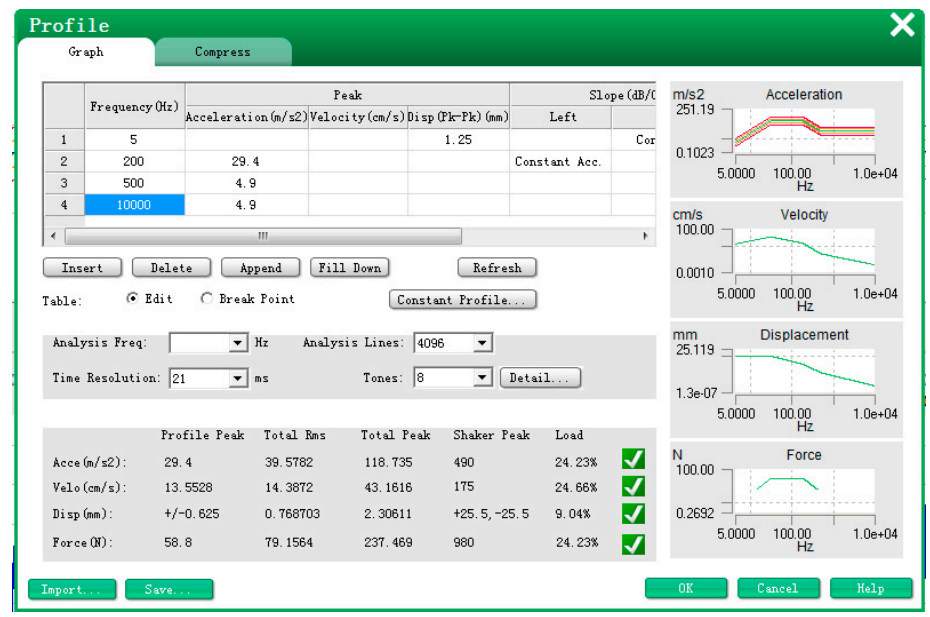

Figure 5. The spectrum settings of composite excitation.
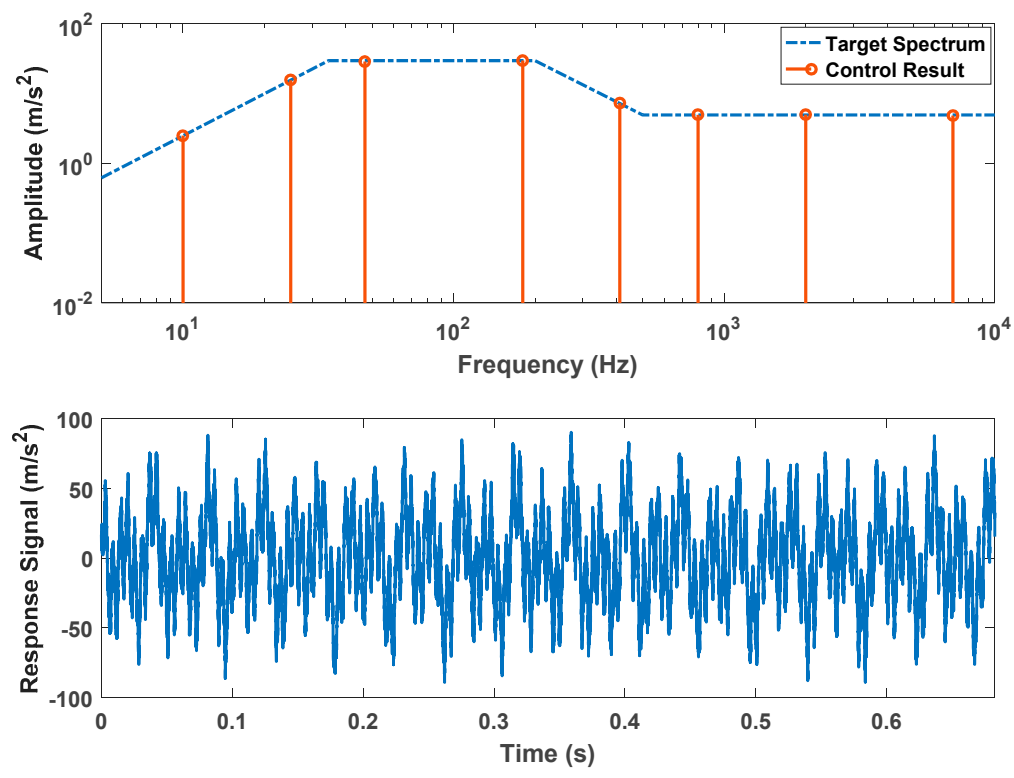

Figure 6. The spectrum and response signal curve of dwell experiment.

Table 1. The control results of eight components in dwell experiment.

\begin{tabular}{|c|c|c|c|c|}
\hline $\begin{array}{c}\text { No. of } \\
\text { Components }\end{array}$ & $\begin{array}{l}\text { Frequency Value } \\
\qquad(\mathrm{Hz})\end{array}$ & $\begin{array}{l}\text { Target Amplitude } \\
\text { Value }\left(\mathrm{m} / \mathrm{s}^{2}\right)\end{array}$ & $\begin{array}{l}\text { Control Result } \\
\qquad\left(\mathrm{m} / \mathrm{s}^{2}\right)\end{array}$ & $\begin{array}{l}\text { Relative } \\
\text { Error (\%) }\end{array}$ \\
\hline 1 & 10.0 & 2.4696 & 2.4794 & 0.40 \\
\hline 2 & 25.0 & 15.4742 & 15.3566 & -0.76 \\
\hline 3 & 47.0 & 29.4000 & 28.4229 & -0.332 \\
\hline 4 & 180.0 & 29.4000 & 29.4686 & 0.23 \\
\hline 5 & 411.0 & 7.1736 & 7.2324 & 0.82 \\
\hline 6 & 800.0 & 4.9000 & 4.9196 & 0.40 \\
\hline 7 & 2000.0 & 4.9000 & 4.9392 & 0.80 \\
\hline 8 & 7000.0 & 4.9000 & 4.9294 & 0.60 \\
\hline
\end{tabular}

\subsection{The Sweeping Experiment of Composite Excitation}

The same spectrum as Section 4.1 was used for the sweeping experiment. The frequency band $5-10,000 \mathrm{~Hz}$ is divided into eight sub-bands, as shown in Table 2 . The eight sub-bands are simultaneously tested at the logarithmic sweep rate of $1 \mathrm{oct} / \mathrm{min}$, wherein for each tracking filter, the passband of the low-pass filter is set to $25 \%$ of the instantaneous target frequency. An instantaneous 
amplitude control result is shown in Figure $7 \mathrm{a}$, on which the ' $\rightarrow$ ' represents the sweep direction. The control result after the sweep experiments completed is shown in Figure $7 \mathrm{~b}$. The maximum relative control error of the whole frequency band does not exceed $5.33 \%$. The experimental results show that the high-precision control of each frequency component was achieved using the technologies presented in this paper.

Table 2. The 8 sub-frequency band settings in sweeping experiment.

\begin{tabular}{cccc}
\hline No. of Frequency Band & Frequency Interval (Hz) & Sweep Rate (Oct/min) & $\begin{array}{c}\text { LP Filter Cutoff } \\
\text { Frequency Factor (\%) }\end{array}$ \\
\hline 1 & $5 \sim 12.93$ & 1 & 25 \\
2 & $12.93 \sim 33.44$ & 1 & 25 \\
3 & $33.44 \sim 86.47$ & 1 & 25 \\
4 & $86.47 \sim 223.61$ & 1 & 25 \\
5 & $223.61 \sim 578.25$ & 1 & 25 \\
6 & $578.25 \sim 1495.35$ & 1 & 25 \\
7 & $1495.35 \sim 3866.97$ & 1 & 25 \\
8 & $3866.97 \sim 10,000$ & & 25 \\
\hline
\end{tabular}
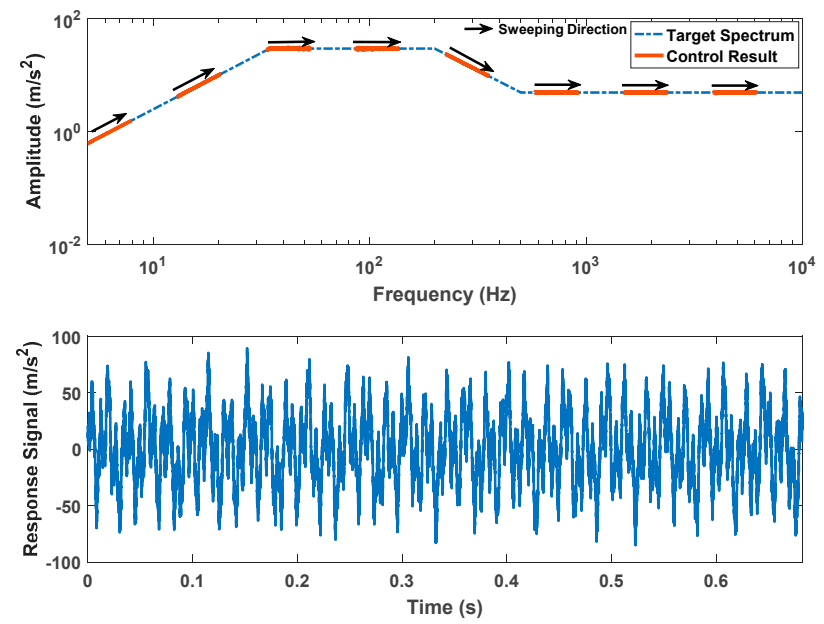

(a)

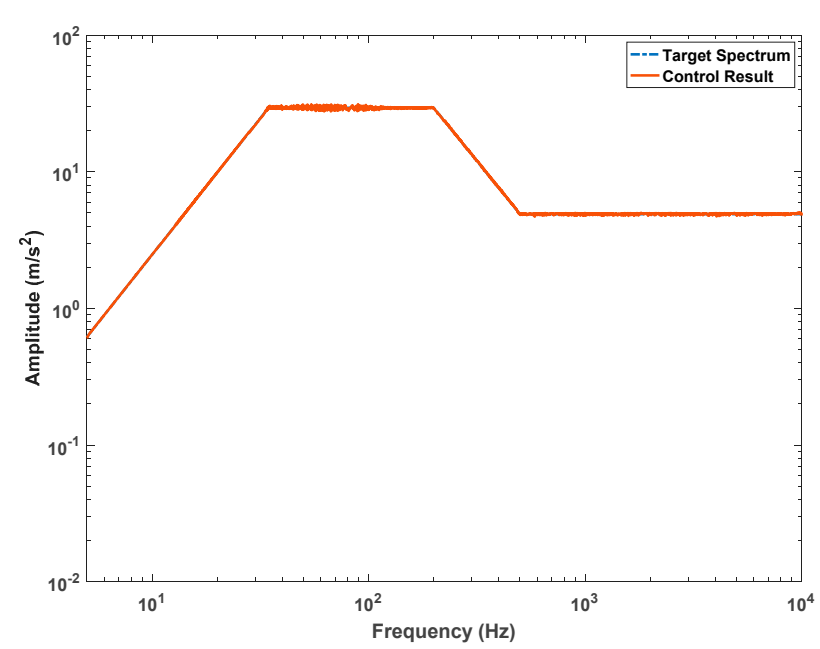

(b)

Figure 7. The results of sweeping experiments: (a) the instantaneous spectrum trajectory and time-domain curve; (b) the spectrum of the sweeping experiment completed. 


\section{Conclusions}

This paper presents a high-precision multi-source vibration simulation technology that is very practical in many areas such as power system health monitoring, faulty diagnosis, vehicle structure modal analysis, noise and vibration, etc. To improve the sinusoidal unsteady state caused by the accumulation of truncations, rounding errors, and the finite word length effect in programming, an explicit phase calculation method is adopted. Furthermore, a parallel tracking filter structure is used and its branches can estimate the amplitude and phase of each frequency component independently. In the tracking filter, cutoff frequency of the low-pass filter can be updated according to the target frequencies dynamically, which makes the tracking filter more tunable and adaptable to various operating conditions. Multi-frequency dwell experiment and multi-frequency sweeping experiment are completed. The experimental results show that the multi-source vibration simulation technologies presented in this paper are very effective in generating excitation signals containing up to eight sinusoidal frequency components. Each frequency component has achieved high-precision amplitude as expected.

Although the technologies presented in this paper have been applied in practice, they also contain problems that may affect further study. If two components $f_{i}$ and $f_{j}$ are closed enough, the first part of Equations (9) and (10) containing $\left(f_{i}-f_{j}\right)$ will be low frequency signals that may introduce errors in steps 3 and 4 in Section 3. It is hard to design low-pass filters to filter out these low frequency (e.g., $1 \mathrm{~Hz}$ ) signals. How to deal with these low frequency signals without slowing down the sweep rate may be a major research direction of multi-source vibration simulation technology in the future.

Author Contributions: Investigation, X.M., Z.C. and H.H.; software, Y.Z.; validation, Z.C.; writing-original draft, X.M.; writing-review and editing, X.M. and Z.C.

Conflicts of Interest: The authors declare no conflict of interest.

\section{References}

1. Li, Z.X.; Jiang, Y.; Hu, C. Recent progress on decoupling diagnosis of hybrid failures in gear transmission systems using vibration sensor signal: A review. Measurement 2016, 90, 4-19. [CrossRef]

2. Glowacz, A. Acoustic based fault diagnosis of three-phase induction motor. Appl. Acoust. 2018, 137, 82-89. [CrossRef]

3. Glowacz, A. Fault diagnosis of single-phase induction motor based on acoustic signals. Mech. Syst. Signal Process. 2019, 117, 65-80. [CrossRef]

4. Li, Z.X.; Jiang, Y.; Hu, C.Q.; Peng, Z.X. Difference equation based empirical mode decomposition with application to separation enhancement of multi-fault vibration signals. J. Differ. Equ. Appl. 2017, 23, $457-467$. [CrossRef]

5. Duan, Z.H.; Wu, T.H.; Guo, S.W.; Shao, T.; Malekian, R.; Li, Z.X. Development and trend of condition monitoring and fault diagnosis of multi-sensors information fusion for rolling bearings: A review. Int. J. Adv. Manuf. Technol. 2018, 96, 803-819. [CrossRef]

6. Xia, W.K.; Li, Z.X.; Tian, Z.; Duan, Z.H. A feature extraction and visualization method for fault detection of marine diesel engines. Measurement 2018, 116, 429-437. [CrossRef]

7. Saucedo-Dorantes, J.J.; Delgado-Prieto, M.; Ortega-Redondo, J.A.; Osornio-Rios, R.A.; Romero-Troncoso, R.d.J. Multiple-fault detection methodology based on vibration and current analysis applied to bearings in induction motors and gearboxes on the kinematic chain. Shock Vib. 2016. [CrossRef]

8. Buzzoni, M.; Mucchi, E.; D’Elia, G.; Dalpiaz, G. Diagnosis of localized faults in multistage gearboxes: A vibrational approach by means of automatic emd-based algorithm. Shock Vib. 2017. [CrossRef]

9. Wang, Z.Y.; Ma, M.X.; Piao, F.X.; Wang, Z.F. The separation of similar frequency sinusoidal signal based on the least square method. J. Electr. Inf. Technol. 2006, 28, 60-65.

10. Yang, Z.D.; Cong, D.C.; Han, J.W.; Li, H.R. Signal synthesis and analysis for swept-sine vibration control. J. Vib. Eng. 2008, 21, 309-311.

11. Yang, M. Study on Parameter Estimation Algorithm for Multiple Sinusoidal Signal under Noisy Background. Master's Thesis, Harbin Engineering University, Harbin, China, 2016. 
12. Sangeetha, P.; Hemamalini, S. Dyadic wavelet transform-based acoustic signal analysis for torque prediction of a three-phase induction motor. IET Signal Process 2017, 11, 604-612. [CrossRef]

13. Zhang, B.Y.; Chen, H.H.; He, X.D. New control method for MIMO swept-sin test. J. Vib. Shock 2015, 34, 199-200.

14. Ning, D.H.; Sun, S.S.; Du, H.P.; Li, W.H.; Li, W.X. Control of a multiple-DOF vehicle seat suspension with roll and vertical vibration. J. Sound. Vib. 2018, 435, 170-191. [CrossRef]

15. Wang, S.C. Research of Real-Time Control System for Vibration Test. Ph.D. Thesis, Zhejiang University, Zhejiang, China, 2006.

16. Rana, K.P.S. Fuzzy control of an electrodynamic shaker for automotive and aerospace vibration testing. Expert. Syst. Appl. 2011, 38, 11335-11346. [CrossRef]

17. Duda, K.; Barczentewicz, S. Interpolated DFT for $\sin \alpha(x)$ Windows. IEEE Trans. Instrum. Meas. 2014, 63, 754-760. [CrossRef]

18. Park, S.Y.; Park, J. A New Four Parameter Estimator of Sampled Sinusoidal Signals without Iteration. IEICE Trans. Fundam. Electr. Commun. Comput. Sci. 2014, E97A, 652-660. [CrossRef]

19. Sun, H.J.; Wang, X.W. Modified rife algorithm for frequency estimation of sinusoid wave based on amplitude and phase criterion. J. Tianjin Univ. Sci. Technol. 2018, 51, 810-813. [CrossRef]

20. Qian, F.Y.; Leung, S.H.; Zhu, Y.S.; Wong, W.; Pao, D.; Lau, W.H. Damped sinusoidal signals parameter estimation in frequency domain. Signal Processing. 2012, 92, 381-391. [CrossRef]

21. Carní, D.L.; Fedele, G. Multi-sine fitting algorithm enhancement for sinusoidal signal characterization. Comput. Stand. Interfaces 2012, 34, 535-540. [CrossRef]

22. Pedro, M.R.; Anónio, C.S. A new sine-fitting algorithm for accurate amplitude and phase measurements in two channel acquisition systems. Measurement 2008, 41, 135-143.

23. Nelson, D. Special purpose correlation functions for improved signal detection and parameter estimation. IEEE Int. Confer. Acoust. 1993, 4, 73-76.

24. Zhu, L.; Shen, J.H. Modified high-lag autocorrelation estimation method for frequency estimation of sinusoidal signal. J. Harbin Eng. Univ. 2016, 37, 579-584.

25. Kuo, S.M.; Gupta, A.; Mallu, S. Development of adaptive algorithm for active sound quality control. J. Sound. Vib. 2007, 299, 12-21. [CrossRef]

26. Liu, J.X.; Zhang, X.W.; Chen, X.F. Modeling and active vibration control of a coupling system of structure and actuators. J. Vib. Control 2016, 22, 382-395. [CrossRef]

27. Liu, J.X. Research on Adaptive Spectral Reshaping Theory of Active Control and Applications. Ph.D. Thesis, Xi'an Jiaotong University, Xi'an, China, 2016. 